\title{
Lycopene supplementation attenuated xanthine oxidase and myeloperoxidase activities in skeletal muscle tissues of rats after exhaustive exercise
}

\author{
Chieh-Chung Liu ${ }^{1}$, Chi-Chang Huang ${ }^{2}$, Wan-Teng Lin ${ }^{3,4}$, Chin-Cheng Hsieh ${ }^{5}$, Shih-Yi Huang ${ }^{2}$, Su-Jiun Lin ${ }^{6}$ and \\ Suh-Ching Yang ${ }^{2} *$ \\ ${ }^{1}$ Department of Physical Education, Yuan Pei University of Science and Technology, Hsin Chu, Taiwan \\ ${ }^{2}$ School of Nutrition and Health Sciences, Taipei Medical University, Taipei, Taiwan \\ ${ }^{3}$ Department of Nutrition and Food Sciences, Fu-Jen Catholic University, Taipei, Taiwan \\ ${ }^{4}$ De Lin Institute of Technology, Taipei, Taiwan \\ ${ }^{5}$ Department of Physical Education, National Hsin-Chu Teacher's College, Hsin Chu, Taiwan \\ ${ }^{6}$ Graduate Institute of Biology and Environment Science, School of Cellular and Molecular Biology, University of New Haven, CT 06516, \\ USA
}

(Received 10 March 2005 - Revised 1 June 2005 - Accepted 7 June 2005)

\begin{abstract}
Strenuous exercise is known to induce oxidative stress leading to the generation of free radicals. The purpose of the present study was to investigate the effects of lycopene, an antioxidant nutrient, at a relatively low dose $(2.6 \mathrm{mg} / \mathrm{kg}$ per d) and a relatively high dose $(7.8 \mathrm{mg} / \mathrm{kg}$ per d) on the antioxidant status of blood and skeletal muscle tissues in rats after exhaustive exercise. Rats were divided into six groups: sedentary control (C); sedentary control with low-dose lycopene (CLL); sedentary control with high-dose lycopene (CHL); exhaustive exercise (E); exhaustive exercise with low-dose lycopene (ELL); exhaustive exercise with high-dose lycopene (EHL). After $30 \mathrm{~d}$, the rats in the three $\mathrm{C}$ groups were killed without exercise, but the rats in the three $\mathrm{E}$ groups were killed immediately after an exhaustive running test on a motorised treadmill. The results showed that xanthine oxidase (XO) activities of plasma and muscle, and muscular myeloperoxidase (MPO) activity in group E were significantly increased compared with group C. Compared with group E, the elevations of XO and MPO activities of muscle were significantly decreased in group EHL. The malondialdehyde concentrations of plasma and tissues in group E were significantly increased by 72 and $114 \%$, respectively, compared with those in group C. However, this phenomenon was prevented in rats of the ELL and EHL groups. There was no significant difference in the GSH concentrations of erythrocytes in each group; however, exhaustive exercise resulted in a significant decrease in the GSH content of muscle. In conclusion, these results suggested that lycopene protected muscle tissue from oxidative stress after exhaustive exercise.
\end{abstract}

Lycopene: Xanthine oxidase: Myeloperoxidase: Oxidative stress: Exhaustive exercise

Recently, strenuous physical sports such as the iron-man triathlon, cross-country biking, and the ultra-marathon are becoming increasing popular around the world (Wu et al. 2004). Physical exercise is characterised by an increase in $\mathrm{O}_{2}$ consumption by the whole body and particularly by muscle. The increase in $\mathrm{O}_{2}$ uptake is associated with a rise in the production of reactive oxygen species. This leads to an increase in lipid peroxidation, and an impairment of both enzymic and non-enzymic antioxidant defence systems of target tissues and blood (Clarkson \& Thompson, 2000). Many studies have indicated that both xanthine oxidase (XO) and myeloperoxidase (MPO) are responsible for exhaustive exercise-induced oxidative stress in several tissues including muscle, liver, and heart. During exhaustive exercise, $\mathrm{XO}$ activity was significantly increased in the circulation and tissues (Westing et al. 1989; Kumar et al. 1992; Radak et al. 1995;
Vina et al. 2000). XO, a metalloflavoprotein, is an important source of oxygen free radicals. It can catalyse the reduction of $\mathrm{O}_{2}$, leading to the formation of superoxide $\left(\mathrm{O}_{2}^{--}\right)$and $\mathrm{H}_{2} \mathrm{O}_{2}$, which has been proposed as a central mechanism of oxidative injury in some situations (McCord, 1985). Tidball (2005) showed that neutrophils rapidly promote muscle damage after exercise. Human and animal studies have also demonstrated that MPO activity in tissue, a marker for neutrophil infiltration, is associated with strenuous exercise-induced tissue damage (Fielding et al. 1993; Belcastro et al. 1996).

Recent research suggests that supplementations of certain antioxidant nutrients are practicable for physically active individuals to recover from tiredness faster and prevent exercise damage (Banerjee et al. 2003). Many studies have indicated that antioxidant nutrient supplementations, such as vitamin $\mathrm{C}$ and vitamin $\mathrm{E}$,

Abbreviations: ALT, alanine aminotransferase; AST, aspartate aminotransferase; C, sedentary control; CHL, sedentary control with a relatively high-dose lycopene; CK, creatine kinase; CLL, sedentary control with a relatively low-dose lycopene; E, exhaustive exercise; EHL, exhaustive exercise with high-dose lycopene; ELL, exhaustive exercise with low-dose lycopene; LDH, lactate dehydrogenase; MDA, malondialdehyde; MPO, myeloperoxidase; UA, uric acid; XO, xanthine oxidase.

* Corresponding author: Dr Suh-Ching Yang, fax +886 22737 3112, email sokei@tmu.edu.tw 
prevented strenuous exercise-induced oxidative injury in human subjects and rats (Kumar et al. 1992; Reddy et al. 1998; Heunks et al. 1999; Khanna et al. 1999; Atalay et al. 2000; Mastaloudis et al. 2004). Lycopene, a carotenoid mostly found in tomatoes and tomato products, is a powerful antioxidant with a singlet-oxygen-quenching capacity greater than that of $\beta$-carotene and vitamin $\mathrm{E}$ by 47 and 100 times, respectively (Di Mascio et al. 1989). However, the role of lycopene in exhaustive exercise-induced oxidative stress in human subjects or animal studies has not been investigated so far. Therefore, the present study has been undertaken with an animal model to investigate the effects of lycopene supplementation on XO and MPO activities in rats after exhaustive exercise. Furthermore, the analysis of lipid peroxidation (malondialdehyde; MDA) and antioxidative substance (GSH) were also performed in the present study.

\section{Materials and methods}

\section{Animals}

The design of the experimental animal model was modified from the studies of Husain (2003). Male Sprague-Dawley rats weighing 260-280g (National Laboratory Animal Breeding and Research Center, National Science Council, Taipei, Taiwan) were used in the present study. All rats were given Purina 5001 powder chow (Rodent Laboratory Chow 5001; Purina, St Louis, MO, USA) and tap water ad libitum and housed in a room maintained at $23 \pm 2{ }^{\circ} \mathrm{C}$ and a $12 \mathrm{~h}$ light-dark cycle. Animals were randomly divided into six groups, such as sedentary control (C), sedentary control with a relatively low-dose lycopene $(2.6 \mathrm{mg} /$ kg per d; CLL), sedentary control with a relatively high-dose lycopene $(7.8 \mathrm{mg} / \mathrm{kg}$ per $\mathrm{d}$; CHL), exhaustive exercise (E), exhaustive exercise with low-dose lycopene (ELL) and exhaustive exercise with high-dose lycopene (EHL). Lycopene was incorporated into the diet as a tomato oleoresin containing $6 \%$ lycopene (Lyco-Red Natural Products Industries Ltd, Beer-Sheva, Israel). The experiment was approved by the Institutional Animal Care and Usage Committee of the Yuan Pei University of Science and Technology and followed the guidelines established by the National Laboratory Animal Breeding and Research Center in Taiwan.

\section{Exercise protocol}

All rats in the three exercise groups were introduced to treadmill running with $15-20 \mathrm{~min}$ exercise bouts at $15-30 \mathrm{~m} / \mathrm{min}$ for $6 \mathrm{~d}$ to accustom them to running. In the formal run, rats warmed up for $15 \mathrm{~min}$ running at the speed of $15 \mathrm{~m} / \mathrm{min}$. Then rats progressed to running for $15 \mathrm{~min}$ at $20 \mathrm{~m} / \mathrm{min}$ and $30 \mathrm{~min}$ at $25 \mathrm{~m} / \mathrm{min}$. Finally, rats ran to exhaustion on the treadmill at a final speed of $30 \mathrm{~m} / \mathrm{min}, 10 \%$ gradient and approximately $70-75 \%$ $\mathrm{VO}_{2} \max$ (Brooks \& White, 1978). The treadmill was equipped with an electric shocking grid on the rear barrier to provide the animal with exercise motivation (T510E treadmill device; DR Instrument, Taipei, Taiwan). The point of exhaustion was determined as when the rat was unable to right itself when placed on its back. To eliminate diurnal effects, the experiments were performed at the same time (09.00-12.00 hours).

\section{Sample preparation}

The rats in the three $\mathrm{C}$ groups were anaesthetised with ethyl ether and killed after $12 \mathrm{~h}$ fasting without exercise. Then, the rats in the exercise groups were killed immediately after exhaustive exercise. Heparinised blood samples were collected from the abdominal aorta, and skeletal muscle tissue was carefully removed, rinsed in ice-cold normal saline, blotted dry and stored at $-80^{\circ} \mathrm{C}$ for further analysis. Blood samples were centrifuged at $1400 \mathrm{~g}$ at $4^{\circ} \mathrm{C}$ for $10 \mathrm{~min}$. The supernatant fractions (plasma) were used for the determination of plasma parameters. The parts of pellets (erythrocytes) were washed three times with icecold saline. The erythrocytes were then re-suspended in four packed cell volumes of ice-cold distilled water to prepare haemolysate. All chemicals used in the present study were purchased from Sigma Chemical Co. (St Louis, MO, USA) unless stated otherwise.

\section{Lycopene analysis}

Plasma and hepatic lycopene were determined with slight modification of a previous study (Lederman et al. 1998). Approximately $1 \mathrm{~g}$ liver was homogenised in $4 \mathrm{ml}$ absolute ethanol (containing $1 \mathrm{~g}$ butylated hydroxytoluene/l) with a tissue homogeniser (Polytron PT3100; Brinkmann Instruments, Littau, Switzerland). Homogenate was saponified by the addition of $1 \mathrm{ml}$ saturated $\mathrm{KOH}$. The mixture was saponified at $70^{\circ} \mathrm{C}$ in a water-bath for $30 \mathrm{~min}$. After the sample was cooled to room temperature, the extract was washed with $2 \mathrm{ml}$ deionised water and extracted three times with $6 \mathrm{ml} n$-hexane. The $n$-hexane layer $(1 \mathrm{ml})$ was evaporated to dryness under vacuum. The residue was re-dissolved in $400 \mu \mathrm{l}$ methanol, and $50 \mu \mathrm{l}$ of sample was subjected to HPLC. The analytical HPLC system for the determination of lycopene concentration was a commercial system (Hitachi Ltd, Tokyo, Japan), including a pump (model L-7100), an autosampler (model L-7200), and a diode array detector (model L-7455; $220-800 \mathrm{~nm}$, set at $470 \mathrm{~nm}$ ). Separations were performed using a $250 \times 4.6 \mathrm{~mm}$, Ace $5 \mathrm{C} 18$ reversed-phase column (ACE-1212546; Advanced Chromatography Technologies, Aberdeen, UK). The mobile-phase mixture of methanol and toluene (3:1, $\mathrm{v} / \mathrm{v})$ was used at a flow rate of $1.5 \mathrm{ml} / \mathrm{min}$. The retention time for the lycopene standard was $5.2 \mathrm{~min}$. Lycopene was estimated as a single peak containing all-trans and cis isomers (Jain et al. 1999). Pure all-trans lycopene was used as an external standard. Quantification of the lycopene was made from measurements of peak areas in relation to standards chromatographed under the same conditions.

\section{Plasma parameters}

Plasma was used for the determination of aspartate aminotransferase (AST), alanine aminotransferase (ALT), lactate dehydrogenase (LDH), creatine kinase (CK), and uric acid (UA) with an automatic analyser (Hitachi 7170; Hitachi Ltd).

\section{The haemoglobin concentration in erythrocyte haemolysates}

In order to express the antioxidant level per $\mathrm{g} \mathrm{Hb}$, total $\mathrm{Hb}$ concentration of erythrocyte haemolysates was measured spectrophotometrically at $540 \mathrm{~nm}$ using a commercial kit (HG 890; Randox Laboratories, County Antrim, UK). 
Total protein concentration in skeletal muscle tissues

In order to express the antioxidant enzymes activities per $g$ protein, total protein concentration of tissue samples was spectrophotometrically estimated according to the method of Lowry et al. (1951) using a Bio-Rad DC protein assay kit (catalogue no. 500-0116; Bio-Rad Laboratories, Hercules, CA, USA).

\section{Xanthine oxidase activities in plasma and skeletal muscle tissues}

$\mathrm{XO}$ activities of plasma and tissue samples were determined by the method of Westerfeld et al. (1959). Diluted sample was added to xanthine $(0.1 \mathrm{mmol} / \mathrm{l}$; dissolved in sodium phosphate buffer $(50 \mathrm{mmol} / \mathrm{l})$; $\mathrm{pH} 7 \cdot 5)$. XO activity was measured at $25^{\circ} \mathrm{C}$ on a Hitachi U-2000 spectrophotometer at $290 \mathrm{~nm}$ for $3 \mathrm{~min}$. A unit of $\mathrm{XO}$ activity was that forming $1 \mu \mathrm{mol}$ urate/min at $25^{\circ} \mathrm{C}$. Activity was expressed in $\mathrm{mU} / \mathrm{ml}$ in plasma and in $\mathrm{U} / \mathrm{g}$ protein for specific activity in tissue samples.

\section{Myeloperoxidase activity in skeletal muscle tissues}

MPO activity of tissue samples was determined as a marker enzyme for measuring neutrophil accumulating in tissue samples, because it is closely correlated with the number of neutrophils present in the tissue (Mullane et al. 1985). MPO activity was determined by the method of Schierwagen et al. (1990). A portion of diluted sample $(50 \mu \mathrm{l})$ was added to $1 \mathrm{ml}$ of mixed substrate containing $\mathrm{H}_{2} \mathrm{O}_{2}$ (3 mmol/l) dissolved in 3,3',3,5'-tetramethylbenzidine (R\&D Systems Inc., Minneapolis, MN, USA). MPO activity was measured at $37^{\circ} \mathrm{C}$ on a Hitachi U-2000 spectrophotometer at $655 \mathrm{~nm}$ for $3 \mathrm{~min}$. One unit of MPO activity was defined arbitrarily as the amount of enzyme necessary to catalyse an increase in absorbance of 1.0 at $655 \mathrm{~nm}$ per min at $37^{\circ} \mathrm{C}$. MPO activity was expressed as $\mathrm{U} / \mathrm{mg}$ protein for specific activity in tissue samples.

\section{Lipid peroxidation in plasma and skeletal muscle tissues}

The MDA concentrations of plasma and tissue samples were assessed colorimetrically at $586 \mathrm{~nm}$ using a commercial kit (Calbiochem 437634; Calbiochem-Novabiochem, La Jolla, CA, USA). Concentration was expressed in $\mu \mathrm{mol} / \mathrm{l}$ in plasma and in $\mu \mathrm{mol} / \mathrm{mg}$ protein in tissue samples.

\section{Reduced glutathione concentrations in erythrocytes and skeletal muscle tissues}

The concentrations of GSH in erythrocyte haemolysates and tissue samples were determined spectrophotometrically at $400 \mathrm{~nm}$ using a commercial kit (Calbiochem 354102; Calbiochem-Novabiochem). Concentration was expressed in $\mu \mathrm{mol} / \mathrm{mg} \mathrm{Hb}$ in haemolysates and in $\mu \mathrm{mol} / \mathrm{mg}$ protein in tissue samples.

\section{Statistical analysis}

Values are expressed as means with their standard errors. To evaluate differences between the groups studied, two-way ANOVA with Fisher's post hoc test was used. SAS software (version 8.2; SAS Institute Inc., Cary, NC, USA) was used to analyse all data. Differences were considered statistically significant when $P<0 \cdot 05$.

\section{Results}

Body weight and endurance time

At the end of $30 \mathrm{~d}$, body weight in groups C, CLL, CHL, E, ELL and EHL were 429 (SEM 6), 416 (SEM 11), 413 (SEM 20), 414 (SEM 10), 421 (SEM 5), and 423 (SEM 9) g, respectively. There was no significant difference in body weight between each group. The mean endurance time of treadmill running to exhaustion was 64 (SEM 4), 65 (SEM 5), and 63 (SEM 5) min, respectively, for groups E, ELL and EHL; there was no significant difference among the three groups.

\section{Plasma and hepatic lycopene concentration}

No lycopene was detected in plasma in all groups. In contrast, hepatic lycopene concentrations in groups CLL, CHL, ELL and EHL were 0.97 (SEM 0.15), 3.58 (SEM 0.59), 0.97 (SEM 0.11), and 3.95 (SEM 0.68) $\mu \mathrm{g} / \mathrm{g}$ liver, respectively, whereas no lycopene was detected in groups $\mathrm{C}$ and $\mathrm{E}$. Hepatic lycopene concentration in group CHL was significantly increased by 2.69 -fold compared with that of group CLL $(P<0 \cdot 05)$. EHL group also showed significantly higher hepatic lycopene content by $3 \cdot 07$-fold, when compared with group ELL $(P<0 \cdot 05)$.

Plasma aspartate aminotransferase, alanine aminotransferase, lactate dehydrogenase, creatine kinase, and uric acid levels

Plasma AST, ALT, LDH, CK, and UA levels in group E were all significantly elevated by $135,58,208,744$ and $413 \%$, respectively, compared with those in group $\mathrm{C}(P<0 \cdot 05)$ (Table 1). However, there was no difference between each group in the plasma level of AST, ALT, LDH, CK, and UA.

Xanthine oxidase activities in plasma and skeletal muscle tissues

As shown in Table 2, XO activities of plasma and muscle in group E were significantly elevated by 35 and $47 \%$, respectively, compared with that in group C. However, the muscular XO activities were significantly lower by 20 and $23 \%$, respectively, in groups ELL and EHL compared with group $\mathrm{E}(P<0 \cdot 05)$.

\section{Myeloperoxidase activity in skeletal muscle tissues}

MPO activity in the skeletal muscle is shown in the Table 2. In comparison with group $\mathrm{C}$, MPO activity of muscle in group $\mathrm{E}$ was significantly higher by $30 \%(P<0 \cdot 05)$. Compared with group E, muscular MPO activity was significantly lower by $27 \%$ in group EHL $(P<0 \cdot 05)$.

\section{Lipid peroxidation in plasma and skeletal muscle tissues}

MDA, a quantitative marker of lipid peroxidation, was measured in plasma and skeletal muscle as shown in Table 3. The MDA levels of plasma and muscle in group $\mathrm{E}$ were significantly higher by 72 and $114 \%$, respectively, compared with group C $(P<0.05)$. Inversely, the extents of the elevations were significantly less in groups ELL and EHL, compared with group E $(P<0 \cdot 05)$. 

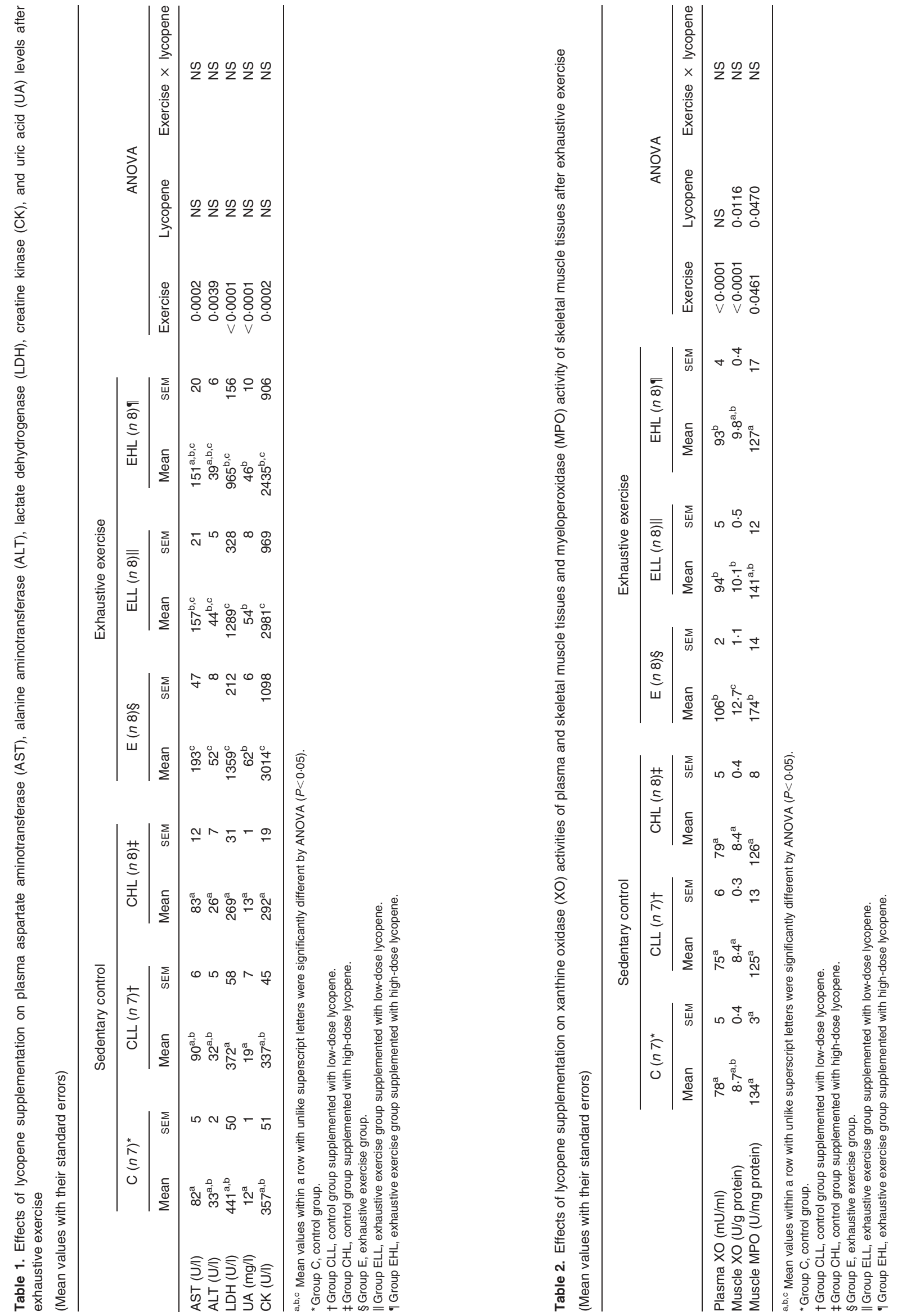
Reduced glutathione concentrations in erythrocytes and skeletal muscle tissues

There was no significant difference in the GSH concentrations of erythrocytes among each group (Table 3). But, muscular GSH content was significantly lower by $34 \%$ in group $\mathrm{E}$ than in group $\mathrm{C}(P<0 \cdot 05)$. No significant differences were observed among group E, ELL, and EHL.

\section{Discussion}

The laboratory rat is a commonly used model for the evaluation of exhaustive exercise effects on biochemical changes in man. The present study aimed to examine lycopene supplementation in relation to exhaustive exercise-induced oxidative injury in a rat model. Hepatic lycopene concentrations increased significantly when rats ingested the lycopene-containing diet in the present study. The present results were consistent with the previous report. This research indicated that lycopene was absorbed and distributed to liver and other tissues (Jain et al. 1999).

\section{Effects of exhaustive exercise and lycopene supplementation on exercise time}

In the present research, no matter whether supplementing lycopene or not, groups E, ELL, and EHL did not have an obvious difference in time of proceeding exercise. The results showed that lycopene supplementation did not influence the performance of exhaustive exercise in rats.

Effects of exhaustive exercise and lycopene supplementation on liver injury

Previous studies have indicated that exhaustive exercise increases AST and ALT activities and results in acute liver damage, including necrosis of the central veins of hepatic lobules, a reduction of hepatocytes number in the microvillus, and a decrease of the endothelium of hepatic veins (Bowers et al. 1978). In the present study, compared with group C, the AST and ALT activities in the plasma of group E were significantly higher by 135 and $58 \%$ respectively (Table 1 ). That is to say, the liver damage might be induced by exhaustive exercise in the present study. This result is also consistent with the previous report by Bowers et al. (1978). However, rats in the lycopene supplementation groups, not only in the CLL and CHL groups but also in the ELL and EHL groups, did not display any differences in AST and ALT activities (Table 1).

Effects of exhaustive exercise and lycopene supplementation on cardiac injury

Much research has indicated that exercise increases plasma LDH activity, which is the index of heart injury. Researchers speculated that free radicals resulting from exercise cause cardiac injury and then LDH is released from cardiocytes into the blood (Itoh et al. 2000). In the present study, compared with group C, the plasma LDH activity of group E was significantly higher, being three times as high (Table 1). This result showed that the acute cardiac injury was induced by exhaustive exercise in this experiment. However, lycopene supplementation did not suppress the elevation of LDH activity in plasma (Table 1). We speculated 
that cardiac injury might not be evaluated only by the plasma LDH activity. Cardiac pathological observation is necessary in the next study.

\section{Effects of exhaustive exercise and lycopene supplementation on hyperuricaemia}

Previous research has indicated that exhaustive exercise increases the $\mathrm{XO}$ activity and further increases the metabolic rate of purine to uric acid in the tissue (Hellsten-Westing et al. 1994; Koyama et al. 1999). Besides, research also reported that exhaustive exercise affects the renal function, resulting in the reduction of nephron glomerular filtration rate and uric acid excretion (Goto et al. 1989). In the present study, the plasma uric acid content in group $\mathrm{E}$ increased by about 4-fold, when compared with group C (Table 2). The result of the research was the same as previous ones and it showed that exhaustive exercise increased uric acid production or decreased uric acid excretion, which led to hyperuricaemia. When rats with exercise were administered lycopene, such as the rats in groups ELL and EHL, the plasma uric acid contents were no different when compared with rats in group E (Table 2). Thus, lycopene supplementation did not inhibit acute hyperuricaemia caused by exhaustive exercise in the present study.

\section{Effects of exhaustive exercise and lycopene supplementation on muscle injury}

In animal experiments, many researchers have already reported that exhaustive exercise elevates CK activity in skeletal muscle. The high CK activity leads to oxidative damage and increases lipid peroxidation in the plasma or muscles significantly (Itoh et al. 2000; Jimenez et al. 2001; Marquez et al. 2001; Zajac et al. 2001). In the present study, compared with group $C$, the plasma CK activity of group E was significantly increased about 7-fold (Table 1), and the MDA contents in the plasma and muscle were also significantly raised 0.8 - and $1 \cdot 11$-fold respectively (Table 3). Inversely, the muscular GSH content was significantly reduced in group E (Table 3 ). The exhaustive exercise caused oxidation damage, increasing lipid peroxidation significantly in the plasma and muscle, and decreasing the contents of antioxidant significantly. Lycopene is a kind of antioxidant. The intake of lycopene-rich food or lycopene supplementation is able to decrease the formation of lipid peroxidation MDA (Pan et al. 2004). In addition, lycopene also inhibits the decrease of GSH in cells (Leal et al. 1999; Gupta et al. 2003). In our research, the supplement of lycopene significantly decreased MDA not only in plasma but also in muscular tissue (Table 3). These results showed that a supplement of lycopene efficiently inhibits lipid peroxidation induced by exhaustive exercise. However, the lycopene supplement did not affect GSH levels, both in erythrocytes and muscle in the present study.

It has been indicated that exhaustive exercise leads to the release of MPO from neutrophils and then induces severe oxidative damage (Belcastro et al. 1996; Suzuki et al. 1996; Yamada et al. 2000; Morozov et al. 2003). MPO is regarded as not only an index of inflammation but also as an index of oxidative damage. In the present study, the muscular MPO activity of group E was obviously higher than that of group C (Table 2). It showed that exhaustive exercise caused muscle injury by increasing the activity of MPO. On the other hand, the activity of MPO in the muscle of group EHL is significantly lower than that of group E (Table 2). Thus, this result indicated that the supplement of lycopene decreased muscular MPO activity after exhaustive exercise. There is only one scientific study that reports decreasing the activity of MPO by lycopene (Reifen et al. 2004), but the mechanism still needs to be investigated in further research.

In conclusion, the present results suggest that: (1) the exhaustive exercise induced oxidative stress and might impair the cardiac, hepatic, renal and muscular functions; (2) lycopene supplementation improved the oxidative damage caused by exhaustive exercise by means of decreasing XO and MPO activities and MDA level in muscular tissue; (3) the non-effectiveness of lycopene supplement on plasma profiles, such as AST, ALT, $\mathrm{LDH}, \mathrm{CK}$, and UA levels, still need further investigation and pathological observation is necessary in a future study, including heart, liver, kidney or muscle.

\section{References}

Atalay M, Laaksonen DE, Khanna S, Kaliste-Korhonen E, Hanninen O \& Sen CK (2000) Vitamin E regulates changes in tissue antioxidants induced by fish oil and acute exercise. Med Sci Sports Exerc 32, 601-607.

Banerjee AK, Mandal A, Chanda D \& Chakraborti S (2003) Oxidant, antioxidant and physical exercise. Mol Cell Biochem 253, 307-312.

Belcastro AN, Arthur GD, Albisser TA \& Raj DA (1996) Heart, liver, and skeletal muscle myeloperoxidase activity during exercise. J Appl Physiol 80, 1331-1335.

Bowers WD Jr, Hubbard RW, Leav I, Daum R, Conlon M, Hamlet MP, Mager M \& Brandt P (1978) Alterations of rat liver subsequent to heat overload. Arch Pathol Lab Med 102, 154-157.

Brooks GA \& White TP (1978) Determination of metabolic and heart rate responses of rats to treadmill exercise. J Appl Physiol 45, 1009-1015.

Clarkson PM \& Thompson HS (2000) Antioxidants: what role do they play in physical activity and health? Am J Clin Nutr 72, 637S-646S.

Di Mascio P, Kaiser S \& Sies H (1989) Lycopene as the most efficient biological carotenoid singlet oxygen quencher. Arch Biochem Biophys 274, 532-538.

Fielding RA, Manfredi T, Ding W, Fiatarone MA, Evans WJ \& Cannon JG (1993) Acute phase response in exercise III. Neutrophil and IL-1 beta accumulation in skeletal muscle. Am J Physiol 265, R166-R172.

Goto H \& Ito A \& Mikami (1989) Effect of exercise on urate exerction. Nippon Seirigaku Zasshi 51, 208-220.

Gupta SK, Trivedi D, Srivastava S, Joshi S, Holder N \& Verma SD (2003) Lycopene alternates oxidative stress induced experimental cataract development: an in vitro and in vivo study. Nutrition 19, 794-799.

Hellsten-Westing Y, Kaijser L, Ekblom B \& Sjodin B (1994) Exchange of purines in human liver and skeletal muscle with short-term exhaustive exercise. Am J Physiol 266, R81-R86.

Heunks LMA, Vina J, van Herwaarden CLA, Folgering HTM, Gimeno A \& Dekhuijzen PNR (1999) Xanthine oxidase is involved in exerciseinduced oxidative stress in chronic obstructive pulmonary disease. Am J Physiol 277, R1697-R1704.

Husain K (2003) Interaction of physical training and chronic nitroglycerin treatment on blood pressure, nitric oxide, and oxidants/antioxidants in the rat heart. Pharmacol Res 48, 253-261.

Itoh H, Ohkuwa T, Yamazaki Y, Shimoda T, Wakayama A, Tamura S, Yamamoto T, Sato Y \& Miyamura M (2000) Vitamin E supplementation attenuates leakage of enzymes following 6 successive days of running training. Int J Sports Med 21, 369-374.

Jain CK, Agarwal S \& Rao AV (1999) The effect of dietary lycopene on bioavailability, tissue distribution, in vivo antioxidant properties and colonic preneoplasia in rats. Nutr Res 19, 1383-1391. 
Jimenez L, Lefevre G, Richard R, Couderc R, Saint George M, Duvallet A \& Rieu M (2001) Oxidative stress in hemodialyzed patients during exhausting exercise. J Sports Med Phys Fitness 41, 513-520.

Khanna S, Atalay M, Laaksonen DE, Gul M, Roy S \& Sen CK (1999) Alpha-lipoic acid supplementation: tissue glutathione homeostasis at rest and after exercise. J Appl Physiol 86, 1191-1196.

Koyama K, Kaya M, Ishigaki T, Tsujita J, Hori S, Seino T \& Kasugai A (1999) Role of xanthine oxidase in delayed lipid peroxidation in rat liver induced by acute exhausting exercise. Eur J Appl Physiol Occup Physiol 80, 28-33.

Kumar CT, Reddy VK, Prasad M, Thyagaraju K \& Reddanna P (1992) Dietary supplementation of vitamin $\mathrm{E}$ protects heart tissue from exercise-induced oxidant stress. Mol Cell Biochem 111, 109-115.

Leal M, Shimada A, Ruiz F \& Gonzalez de Mejia E (1999) Effect of lycopene on lipid peroxidation and glutathione-dependent enzymes induced by T-2 toxin in vivo. Toxicol Lett 109, 1-10.

Lederman JD, Overton KM, Hofmann NE, Moore BJ, Thornton J \& Erdman JW (1998) Ferrets (Mustela putoius furo) inefficiently convert $\beta$-carotene to vitamin A. J Nutr 128, 271-279.

Lowry OH, Rosebrough NJ, Lewis Farr A \& Randall RJ (1951) Protein measurement with the Folin phenol reagent. J Biol Chem 193, 265-275.

McCord JM (1985) Oxygen-derived free radicals in postischemic tissue injury. N Engl J Med 312, 159-163.

Marquez R, Santangelo G, Sastre J, Goldschmidt P, Luyckx J, Pallardo FV \& Vina J (2001) Cyanoside chloride and chromocarbe diethylamine are more effective than vitamin $\mathrm{C}$ against exercise-induced oxidative stress. Pharmacol Toxicol 89, 255-258.

Mastaloudis A, Morrow JD, Hopkins DW, Devaraj S \& Traber MG (2004) Antioxidant supplementation prevents exercise-induced lipid peroxidation, but not inflammation, in ultramarathon runners. Free Radic Biol Med 36, 1329-1341.

Morozov VI, Pryatkin SA, Kalinski MI \& Rogozkin VA (2003) Effect of exercise to exhaustion on myeloperoxidase and lysozyme release from blood neutrophils. Eur J Appl Physiol 89, 257-262.

Mullane KM, Kraemer R \& Smith B (1985) Myeloperoxidase activity as a quantitative assessment of neutrophil infiltration into ischemic myocardium. J Pharmacol Methods 14, 157-167.

Pan H, Jiang X, Wan L, Na L \& Wang J (2004) Experimental studies of lycopene in inhibiting tumor growth in S180-bearing mice. Wei Sheng Yап Јіи 33, 456-457.
Radak Z, Asano K, Inoue M, Kizaki T, Oh-Ishi S, Suzuki K, Taniguchi N \& Ohno H (1995) Superoxide dismutase derivative reduces oxidative damage in skeletal muscle of rats during exhaustive exercise. $J$ Appl Physiol 79, 129-135.

Reddy KV, Kumar TC, Prasad M \& Reddanna P (1998) Pulmonary lipid peroxidation and antioxidant defenses during exhaustive physical exercise: the role of vitamin $\mathrm{E}$ and selenium. Nutrition 14, 448-451.

Reifen R, Nissenkorn A, Matas Z \& Bujanover Y (2004) 5-ASA and lycopene decrease the oxidative stress and inflammation induced by iron in rats with colitis. J Gastroenterol 39, 514-519.

Schierwagen C, Bylund-Fellenius AC \& Lundberg C (1990) Improved method for quantification of tissue PMN accumulation measured by myeloperoxidase activity. J Pharmacol Methods 23, 179-186.

Suzuki K, Sato H, Kikuchi T, Abe T, Nakaji S, Sugawara K, Totsuka M, Sato K \& Yamaya K (1996) Capacity of circulating neutrophils to produce reactive oxygen species after exhaustive exercise. J Appl Physiol 81, $1213-1222$.

Tidball JG (2005) Inflammatory processes in muscle injury and repair. Am J Physiol 288, R345-R353.

Vina J, Gimeno A, Sastre J, Desco C, Asensi M, Pallardo FV, Cuesta A, Ferrero JA, Terada LS \& Repine JE (2000) Mechanism of free radical production in exhaustive exercise in humans and rats; role of xanthine oxidase and protection by allopurinol. IUBMB Life $\mathbf{4 9}$, 539-544.

Westerfeld WW, Richert DA \& Higgins ES (1959) Further studies with xanthine oxidase inhibitors. J Biol Chem 234, 1897-1900.

Westing YH, Ekblom B \& Sjodin B (1989) The metabolic relation between hypoxanthine and uric acid in man following maximal shortdistance running. Acta Physiol Scand 137, 341-345.

Wu HJ, Chen KT, Shee BW, Chang HC, Huang YJ \& Yang RS (2004) Effects of $24 \mathrm{~h}$ ultra-marathon on biochemical and hematological parameters. World J Gastroenterol 10, 2711-2714.

Yamada M, Suzuki K, Kudo S, Totsuka M, Simoyama T, Nakaji S \& Sugawara K (2000) Effect of exhaustive exercise on human neutrophils in athletes. Luminescence 15, 15-20.

Zajac A, Waskiewicz Z \& Pilis W (2001) Anaerobic power, creatine kinase activity, lactate concentration, and acid-base equilibrium changes following bouts of exhaustive strength exercises. J Strength Cond Res 15, 357-361. 